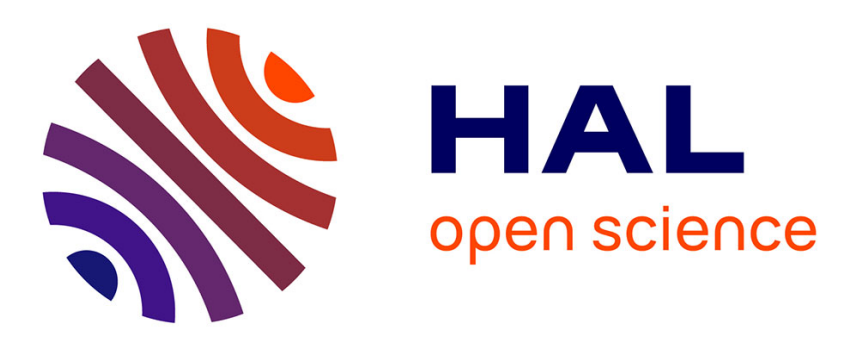

\title{
A perturbation-method-based a posteriori estimator for the planewave discretization of nonlinear Schrödinger equations
}

Eric Cancès, Geneviève Dusson, Yvon Maday, Benjamin Stamm, Martin Vohralík

\section{To cite this version:}

Eric Cancès, Geneviève Dusson, Yvon Maday, Benjamin Stamm, Martin Vohralík. A perturbationmethod-based a posteriori estimator for the planewave discretization of nonlinear Schrödinger equations. Comptes Rendus. Mathématique, 2014, 352 (11), pp.941-946. 10.1016/j.crma.2014.09.014 . hal-00994568

\section{HAL Id: hal-00994568 \\ https://hal.science/hal-00994568}

Submitted on 21 May 2014

HAL is a multi-disciplinary open access archive for the deposit and dissemination of scientific research documents, whether they are published or not. The documents may come from teaching and research institutions in France or abroad, or from public or private research centers.
L'archive ouverte pluridisciplinaire HAL, est destinée au dépôt et à la diffusion de documents scientifiques de niveau recherche, publiés ou non, émanant des établissements d'enseignement et de recherche français ou étrangers, des laboratoires publics ou privés. 


\title{
A perturbation-method-based a posteriori estimator for the planewave discretization of nonlinear Schrödinger equations
}

\author{
Eric Cancès ${ }^{1}$, Geneviève Dusson ${ }^{2,3}$, Yvon Maday ${ }^{2,4}$, Benjamin Stamm², \\ Martin Vohralík ${ }^{5}$, \\ 1 - Université Paris Est, CERMICS, Ecole des Ponts and INRIA, 6 \& 8 Av. Pascal, 77455 Marne-la-Vallée, France \\ 2 - Sorbonne Universités, UPMC Univ. Paris 06 and CNRS, UMR 7598, Laboratoire Jacques-Louis Lions, F-75005, \\ Paris, France \\ 3 - UPMC Univ. Paris 06, Institut du Calcul et de la Simulation, F-75005, Paris, France \\ 4 - Institut Universitaire de France, F-75005, Paris, France, and Division of Applied Mathematics, Brown University, \\ 182 George St, Providence, RI 02912, USA \\ 5 - INRIA Paris-Rocquencourt, Domaine de Voluceau - Rocquencourt, B.P. 105, 78153 Le Chesnay, France
}

\begin{abstract}
In this Note, we propose a new method, based on perturbation theory, to post-process the planewave approximation of the eigenmodes of periodic Schrödinger operators. We then use this post-processing to construct an accurate a posteriori estimator for the approximations of the (nonlinear) Gross-Pitaevskii equation, valid at each step of a self-consistent procedure. This allows us to design an adaptive algorithm for solving the Gross-Pitaevskii equation, which automatically refines the discretization along the convergence of the iterative process, by means of adaptive stopping criteria.

\section{Résumé}

Dans cette Note, nous proposons une nouvelle méthode, basée sur la théorie des perturbations, pour post-traiter l'approximation dans une base d'ondes planes des modes propres d'opérateurs de Schrödinger périodiques. Nous utilisons ensuite ce post-traitement pour construire un estimateur d'erreur a posteriori pour les approximations de l'équation de Gross-Pitaevskii (non-linéaire), valide à chaque étape de la procédure auto-cohérente. Ceci nous permet de proposer un algorithme adaptatif pour résoudre cette équation, qui raffine automatiquement la discrétisation au cours du processus itératif, par le biais de critères d'arrêt adaptatifs.
\end{abstract}

\section{Introduction}

The time-independent Gross-Pitaevskii and Kohn-Sham models are widely used in quantum physics to describe, respectively, steady states of Bose-Einstein condensates and electronic ground states of molecular systems. Although these models dramatically differ from the physical viewpoint (the former describes bosons, the latter describes fermions), their mathematical structures share some similarities: they are constrained optimization problems whose Euler-Lagrange equations are nonlinear elliptic eigenvalue problems. As a consequence, the numerical methods used to simulate these models have common features. In both cases, numerical solutions can be obtained either by minimization of the energy functional on the admissible set, or by solving the Euler-Lagrange equations by a self-consistent field (SCF) procedure. Algorithms mixing the two approaches have also been proposed.

Among the various discretization methods available, the planewave (Fourier) method is one of the most popular. It is in particular the state-of-the-art method for Kohn-Sham simulations in solid state

\footnotetext{
Email addresses: cances@cermics.enpc.fr (Eric Cancès ${ }^{1}$ ), dusson@ann.jussieu.fr (Geneviève Dusson ${ }^{2,3}$ ), maday@ann.jussieu.fr (Yvon Maday ${ }^{2,4}$ ), stamm@ann.jussieu.fr (Benjamin Stamm ${ }^{2}$ ), martin.vohralik@inria.fr (Martin Vohralík ${ }^{5}$.
}

Preprint submitted to Elsevier Science 
physics and materials science. This method consists in imposing periodic conditions at the boundary of the simulation domain $\Omega$ (a cubic box, or more generally the unit cell of a periodic lattice $\mathcal{R} \subset \mathbb{R}^{d}$ ), and in expanding the wave function (or more precisely the occupied orbitals in the Kohn-Sham setting) in the discretization space

$$
X_{N}:=\operatorname{Span}\left(e_{k},|k| \leq N\right),
$$

where $e_{k}(x):=|\Omega|^{-1} e^{i k \cdot x}$ is the Fourier mode with wavevector $k \in \mathcal{R}^{*}, \mathcal{R}^{*}$ denoting the dual lattice of $\mathcal{R}$. For example, we have for a cubic lattice, $\Omega=(0, L)^{d}, \mathcal{R}=L \mathbb{Z}^{d}$, and $\mathcal{R}^{*}=\frac{2 \pi}{L} \mathbb{Z}^{d}$. Let the periodic Lebesgue space $L_{\#}^{2}(\Omega)$ and the periodic Sobolev spaces $H_{\#}^{r}(\Omega)(r \in \mathbb{R})$ be defined by

$$
\begin{aligned}
& L_{\#}^{2}(\Omega):=\left\{v \in L_{\mathrm{loc}}^{2}\left(\mathbb{R}^{d}\right) \mid v \mathcal{R} \text {-periodic }\right\}=\left\{v=\left.\sum_{k \in \mathcal{R}^{*}} \widehat{v}_{k} e_{k}\left|\|v\|_{L_{\#}^{2}}^{2}:=\sum_{k \in \mathcal{R}^{*}}\right| \widehat{v}_{k}\right|^{2}<\infty\right\}, \\
& H_{\#}^{r}(\Omega):=\left\{v=\left.\sum_{k \in \mathcal{R}^{*}} \widehat{v}_{k} e_{k}\left|\|v\|_{H_{\#}^{r}}^{2}:=\sum_{k \in \mathcal{R}^{*}}\left(1+|k|^{2}\right)^{r}\right| \widehat{v}_{k}\right|^{2}<\infty\right\} .
\end{aligned}
$$

We denote by $\Pi_{N}$ the operator on the space of $\mathcal{R}$-periodic tempered distributions defined by

$$
\Pi_{N}\left(\sum_{k \in \mathcal{R}^{*}} \widehat{v}_{k} e_{k}\right)=\sum_{|k| \leq N} \widehat{v}_{k} e_{k}
$$

$\left(\left.\Pi_{N}\right|_{H_{\#}^{s}(\Omega)}\right.$ is in fact the orthogonal projector from $H_{\#}^{s}(\Omega)$ to $X_{N}$ for any $\left.s \in \mathbb{R}\right)$, and by $\Pi_{N}^{\perp}=\left(1-\Pi_{N}\right)$ the orthogonal projector on the orthogonal space $X_{N}^{\perp}$ of $X_{N}$ in $H_{\#}^{s}(\Omega)$ (for any $s \in \mathbb{R}$ ). The periodic Gross-Pitaevskii model reads

$$
\inf \left\{E_{\mathrm{GP}}(v), v \in H_{\#}^{1}(\Omega),\|v\|_{L_{\#}^{2}}=1\right\},
$$

where the Gross-Pitaevskii functional is given, in absence of an external magnetic field, by

$$
E_{\mathrm{GP}}(v):=\int_{\Omega}|\nabla v|^{2}+\int_{\Omega} V|v|^{2}+\frac{\mu}{2} \int_{\Omega}|v|^{4}
$$

where $V$ is a real-valued periodic potential belonging to $H_{\#}^{s}(\Omega)$ for some $s>d / 2$, and $\mu \in \mathbb{R}$. The physical setting corresponds to $d=3$, but our analysis is valid for any dimension $d \leq 3$. On the other hand, we will assume for simplicity that the real parameter $\mu$, which models the magnitude of the interactions between the condensed particles is non-negative (repulsive interactions). It is well-known that (1) has a unique minimizer $u$ up to a global phase, and that (still up to a global phase) $u$ is real-valued, belongs to $H_{\#}^{2}(\Omega)$, and is everywhere positive. In addition, the function $u$ satisfies the Euler-Lagrange equation

$$
-\Delta u+V u+\mu u^{3}=\lambda u, \quad\|u\|_{L_{\#}^{2}}=1,
$$

where $\lambda$, the Lagrange multiplier of the constraint $\|v\|_{L_{\#}^{2}}=1$, is in fact the lowest eigenvalue of the meanfield operator $H_{u}:=-\Delta+V+\mu u^{2}$. Note that $H_{u}$ is a bounded-below self-adjoint operator on $L_{\#}^{2}(\Omega)$ with domain $H_{\#}^{2}(\Omega)$ and compact resolvent. The variational approximation of $(1)$ in the discretization space $X_{N}$ gives rise to the finite-dimensional constrained optimization problem

$$
\inf \left\{E_{\mathrm{GP}}\left(v_{N}\right), v_{N} \in X_{N},\left\|v_{N}\right\|_{L_{\#}^{2}}=1\right\} .
$$

As $\Pi_{N}$ and the Laplace operator commute, the minimizer $\lambda_{N}$ of (2) satisfies

$$
-\Delta u_{N}+\Pi_{N}\left(\left(V+\left|u_{N}\right|^{2}\right) u_{N}\right)=\lambda_{N} u_{N}, \quad\left\|u_{N}\right\|_{L_{\#}^{2}}=1
$$

where $\lambda_{N}$ is the Lagrange multiplier associated with the constraint $\left\|v_{N}\right\|_{L_{\#}^{2}}=1$. It is proved in [2] that, for $N$ large enough, the discretized problem (2) has a unique real-valued minimizer $u_{N}$ such that 
$\left(u, u_{N}\right)_{L_{\#}^{2}} \geq 0$ (the other minimizers being equal to $u_{N}$ up to a global phase), and that the following optimal a priori error estimates hold

$$
\begin{aligned}
c\left\|u-u_{N}\right\|_{H_{\#}^{1}}^{2} & \leq E_{\mathrm{GP}}\left(u_{N}\right)-E_{\mathrm{GP}}(u) \leq C\left\|u-u_{N}\right\|_{H_{\#}^{1}}^{2}, \\
\left|\lambda-\lambda_{N}\right| & \leq C N^{-2(s+1)}, \quad\left\|u-u_{N}\right\|_{H_{\#}^{r}} \leq C N^{-(s+2-r)},
\end{aligned}
$$

for all $-s \leq r<s+2$, and where the constants $c$ and $C$ depend on $\|V\|_{H_{\#}^{s}}$ and $\mu$ but are independent of $N$.

As mentioned above, the discretized problem (3) can be solved either by constrained minimization or by SCF algorithms. Our approach can be applied to a variety of iteration schemes, but for brevity, we only discuss here the simple case of the Roothaan algorithm. We are aware that the Roothaan algorithm is far from being the most efficient algorithm to solve the Gross-Pitaevskii equation, but we nevertheless chose it for two reasons: first, it allows us to illustrate our approach on a simple example where technicalities are reduced to a minimum, and second this algorithm is somewhat similar to the SCF algorithms actually used in Kohn-Sham simulations.

The purpose of this Note is twofold. First, we propose a method to post-process the planewave approximation of the eigenmodes of (linear) periodic Schrödinger operators and obtain a much better accuracy for a limited extra computational cost. This post-processing, although based on a simple application of the perturbation method, seems to be new. Note that this approach can be extended to the case of the (nonlinear) Gross-Pitaevski equation [3]. Second, we use this post-processing to construct an accurate $a$ posteriori error estimator for the Gross-Pitaevskii equation. This estimator allows us to design an adaptive algorithm for solving this equation, which automatically refines the discretization along the convergence of the SCF iterations, by means of adaptive stopping criteria. The first steps of the SCF procedure are carried out in a coarse discretization space (it is indeed a loss of time to use a fine discretization space far from the minimizer), and the cut-off $N$ is gradually increased to balance the two components of the total error: the discretization error on the one hand, and the error due to the fact that the SCF iterations has not converged yet on the other hand. The extension of this approach to more complex nonlinear Schrödinger equations and to the Kohn-Sham model is work in progress.

\section{Post-processing of the planewave approximation of periodic Schrödinger operators}

We first consider a generic linear periodic Schrödinger operator $H=-\Delta+W$ on $L_{\#}^{2}(\Omega)$, with $W \in$ $H_{\#}^{s}(\Omega)$ for some $s \geq 0$. In regard of the previous section, $H$ can denote the linearized Gross-Pitaevskii Hamiltonian $H_{u^{j-1}}=-\Delta+V+\mu\left(u^{j-1}\right)^{2}$ at step $j$ around an approximation $u^{j-1}$ for example. For simplicity, we focus on the ground state eigenvalue $\nu$ of $H$, but our argument straightforwardly extends to any non-degenerate eigenvalues by using the minmax principle. The case of degenerate eigenvalue is dealt with in [3]. Denoting by $w \in H_{\#}^{2}(\Omega)$ the unique positive ground state of $H$, it is well-known that the optimization problem

$$
\nu_{N}=\inf \left\{E_{\mathrm{S}}\left(v_{N}\right), v_{N} \in X_{N},\left\|v_{N}\right\|_{L_{\#}^{2}}=1\right\} \quad \text { with } \quad E_{\mathrm{S}}(v):=\int_{\Omega}|\nabla v|^{2}+\int_{\Omega} W|v|^{2}=(v, H v)_{L_{\#}^{2}}
$$

has a unique real-valued minimizer $w_{N}$ such that $\left(w_{N}, 1\right)_{L_{\#}^{2}} \geq 0$, and that (focusing on the $H^{1}$-norm for brevity)

$$
c\left\|w-w_{N}\right\|_{H_{\#}^{1}}^{2} \leq \nu_{N}-\nu \leq C\left\|w-w_{N}\right\|_{H_{\#}^{1}}^{2} \quad \text { and } \quad\left\|w-w_{N}\right\|_{H_{\#}^{1}} \leq C N^{-(s+1)},
$$

with $0<c \leq C<\infty$ constants independent of $N$. The observation our approach is based upon is that $\left(\nu_{N}, w_{N}\right)$ satisfies 


$$
H_{N} w_{N}=\nu_{N} w_{N},\left\|w_{N}\right\|_{L_{\#}^{2}}=1 \quad \text { where } \quad H_{N}=-\Delta+\Pi_{N} W \Pi_{N},
$$

while $(\nu, w)$ satisfies

$$
\left(H_{N}+\mathcal{W}_{N}\right) w=\nu w,\|w\|_{L_{\#}^{2}}=1 \quad \text { with } \quad \mathcal{W}_{N}=\Pi_{N}^{\perp} W \Pi_{N}+\Pi_{N} W \Pi_{N}^{\perp}+\Pi_{N}^{\perp} W \Pi_{N}^{\perp} .
$$

In addition, it can be seen that as soon as $N^{2}>\nu_{N}, w_{N}$ is the ground state of $H_{N}$. It therefore makes sense to apply Kato's perturbation theory using $\left(\nu_{N}, w_{N}\right)$ as the reference solution and $(\nu, w)$ as the perturbed solution, in order to build improved approximations $\left(\widetilde{\nu}_{N}, \widetilde{w}_{N}\right)$ based upon perturbation arguments. A simple calculation shows that the first order correction to the eigenfunction $w_{N}$ is given by

$$
w_{N}^{(1)}=-\left(-\left.\Delta\right|_{X_{N}^{\perp}}-\nu_{N}\right)^{-1} r_{N} \quad \text { where } \quad r_{N}=H w_{N}-\nu_{N} w_{N}=\Pi_{N}^{\perp}\left(W w_{N}\right) \in X_{N}^{\perp}
$$

is the residual of the eigenvalue problem. The first order correction to the eigenvalue vanishes and the second order correction is (using Dirac's bra-ket notation)

$$
\nu_{N}^{(2)}=\left(w_{N}^{(1)}, r_{N}\right)_{L_{\#}^{2}}=-\left\langle r_{N}\left|\left(-\left.\Delta\right|_{X_{N}^{\perp}}-\nu_{N}\right)^{-1}\right| r_{N}\right\rangle .
$$

Note that $w_{N}^{(1)}$ and $\nu_{N}^{(2)}$ are well-defined (provided that $N^{2}>\nu_{N}$ ) and computable by a single Fast Fourier Transform on a finer grid. An extension to the result below to more general operators and discretization spaces, higher-order perturbations, and degenerate eigenvalues, is proved in [3].

Lemma 2.1 ([3]) Let $W \in H_{\#}^{s}(\Omega)$ for some $s>d / 2$. For all $N \in \mathbb{N}$ such that $N^{2}>\nu_{N}$, the quantities $\widetilde{w}_{N}=w_{N}+w_{N}^{(1)}$ and $\widetilde{\nu}_{N}=\nu_{N}+\nu_{N}^{(2)}$ are well-defined and there exists a constant $C>0$ independent of $N$ such that

$$
\left|\nu-\widetilde{\nu}_{N}\right| \leq C N^{-2}\left|\nu-\nu_{N}\right| \quad \text { and } \quad\left\|w-\widetilde{w}_{N}\right\|_{H_{\#}^{1}} \leq C N^{-2}\left\|w-w_{N}\right\|_{H_{\#}^{1}} .
$$

\section{A posteriori estimators and adaptive algorithms for the Gross-Pitaevskii equation}

We now use the results established in the previous section to design an adaptive algorithm based on the Roothaan procedure for the solution of (3), whose iterations are defined by:

$$
-\Delta u^{j}+\Pi_{N_{j}}\left(V u^{j}+\mu\left|u^{j-1}\right|^{2} u^{j}\right)=\lambda^{j} u^{j}, \quad u^{j} \in X_{N_{j}}, \quad\left\|u^{j}\right\|_{L_{\#}^{2}}=1, \quad\left(u^{j}, 1\right)_{L_{\#}^{2}} \geq 0,
$$

where $u^{0} \in X_{N_{0}}$ is a given initial guess, and where $\left(N_{j}\right)_{j \in \mathbb{N}}$ is a non-decreasing sequence of integers. Note that $u^{j}$ is in some sense a shortcut notation for $u_{N_{j}}^{j}$, since this function belongs to the discrete space $X_{N_{j}}$. Usually, $N_{j}$ is kept constant (equal to $N$ ) along the iterations. In our approach, the sequence $\left(N_{j}\right)_{j \in \mathbb{N}}$ is not specified a priori, but automatically constructed by the algorithm. This is achieved by using an a posteriori estimator of the error between $u^{j}$ and $u$, which can be decomposed into two non-negative contributions:

i) a contribution $\eta_{\mathrm{scf}}^{j}$ measuring the error at step $j$ due to the fact that the SCF procedure has not converged;

ii) a contribution $\eta_{\text {dis }}^{j}$ measuring the error at step $j$ due to the fact that $u^{j}$ is a finite-dimensional approximation of $u$ in $X_{N_{j}}$.

Following the ideas of $[1,4,5]$, we propose to refine the discretization space whenever $\eta_{\mathrm{scf}}^{j} \leq \zeta \eta_{\mathrm{dis}}^{j}$, where $\zeta$ is some user-given parameter, typically of order 0.1 . The new discretization parameter $N_{j+1}$ can then be chosen by linear regression based on the a priori convergence rate in order that the predicted new discretization error is smaller than $\zeta^{\prime} \eta_{\text {scf }}^{j}$ for some user-given parameter $\zeta^{\prime}$. 
Let us now provide explicit expressions for $\eta_{\mathrm{scf}}^{j}$ and $\eta_{\mathrm{dis}}^{j}$. As usual in a posteriori error estimations, we begin with discussing the choice of the measure of the error. A common choice for nonlinear problems is the energy difference $E_{\mathrm{GP}}\left(u^{j}\right)-E_{\mathrm{GP}}(u)$. In our setting, it appears that we can better estimate the augmented energy difference $J_{u}\left(u^{j}\right)$ where

$$
J_{u}(v):=E_{\mathrm{GP}}(v)-E_{\mathrm{GP}}(u)+\frac{\mu}{2} \int_{\Omega}\left(|u|^{2}-|v|^{2}\right)^{2} .
$$

Note that for all $v \in H_{\#}^{1}(\Omega)$ such that $\|v\|_{L_{\#}^{2}}=1$ and $(v, 1)_{L_{\#}^{2}} \geq 0, J(v) \geq 0$ and $J(v)=0$ if and only if $v=u$. We also know from [2] that there exists $0<c \leq C<\infty$ such that $c\|v-u\|_{H_{\#}^{1}}^{2} \leq J_{u}(v) \leq$ $C\|v-u\|_{H_{\#}^{1}}^{2}$ for all $v \in H_{\#}^{1}(\Omega)$ such that $\|v\|_{L_{\#}^{2}}=1$ and $(v, 1)_{L_{\#}^{2}} \geq 0$.

Theorem 3.1 ([3]) Let $u$ be the exact solution of (1) satisfying $u>0$ in $\Omega$ and $u^{j} \in X_{N_{j}}$ the numerical approximation of $u$ generated by the iterative procedure (5) at step $j$. Then

$$
J_{u}\left(u^{j}\right) \leq \eta_{\mathrm{tot}}^{j}:=\left(\lambda^{j}-\lambda_{\mathrm{lb}}^{j+1}+\int_{\Omega}\left[\left(u^{j}\right)^{2}-\left(u^{j-1}\right)^{2}\right]\left(u^{j}\right)^{2}\right),
$$

where $\lambda_{1 \mathrm{~b}}^{j+1}$ is a lower bound of the exact ground state eigenvalue $\lambda_{\infty}^{j+1}$ of $H_{u^{j}}$. In addition, the error bound $\eta_{\mathrm{tot}}^{j}$ can be decomposed as $\eta_{\mathrm{tot}}^{j}=\eta_{\mathrm{scf}}^{j}+\eta_{\mathrm{dis}}^{j}$ where

$$
\eta_{\mathrm{dis}}^{j}:=\left(\lambda_{N_{j}}^{j+1}-\lambda_{\mathrm{lb}}^{j+1}\right) \geq 0, \quad \eta_{\mathrm{scf}}^{j}:=\left(\int_{\Omega}\left[\left(u^{j}\right)^{2}-\left(u^{j-1}\right)^{2}\right]\left(u^{j}\right)^{2}+\lambda^{j}-\lambda_{N_{j}}^{j+1}\right) \geq 0,
$$

where $\lambda_{N_{j}}^{j+1}$ is the variational approximation of $\lambda_{\infty}^{j+1}$ in the discretization space $X_{N_{j}}$.

In the numerical results reported in the next section, we first compute $\lambda_{N_{j}}^{j+1}$ as the lowest eigenvalue of $-\Delta+\Pi_{N_{j}} W \Pi_{N_{j}}$ with $W=V+\mu\left|u^{j}\right|^{2}$ (which amounts to make one more iteration of the Roothaan algorithm in the discretization space $X_{N_{j}}$ ), and we estimate $\lambda_{\infty}^{j+1}$ by post-processing $\lambda_{N_{j}}^{j+1}$ thanks to the perturbation argument provided in Section 2. Although the so-obtained post-processed eigenvalue is not strictly speaking a lower bound of $\lambda_{\infty}^{j+1}$, it is an excellent approximation of it and can be therefore be used instead of $\lambda_{\mathrm{lb}}^{j+1}$ to compute the error estimators in Theorem 3.1. Note that it is also possible to use perturbation theory to estimate both $\lambda_{N_{j}}^{j+1}$ and $\lambda_{\mathrm{lb}}^{j+1}$ (see [3]).

\section{Numerical Results}

We first consider the linear one-dimensional periodic Schrödinger operator with potential $W(x)=$ $|\sin (2 \pi x)|+6$ and $\Omega=(0,1)$. We observe in Fig. 1 (left) very good agreements with the theoretical results of Lemma 2.1. We then report in Fig. 1 (right) simulations of the one-dimensional Gross-Pitaevskii equation with $V(x)=|\sin (2 \pi x)|+6$ and $\mu=1$, showing the decay of different quantities related to Theorem 3.1 for a fixed $N=50$ during the Roothaan iterations, and illustrating the sharpness of the estimator and the splitting of the error components. More extensive numerical results in higher dimensions will be presented in [3].

Acknowledgements. This work was partially undertaken in the framework of CALSIMLAB, supported by the public grant ANR-11-LABX-0037-01 overseen by the French National Research Agency (ANR) as part of the Investissements dAvenir program (reference : ANR-11-IDEX-0004-02). Financial support by the ANR grants Manif and Becasim is also acknowledged. 

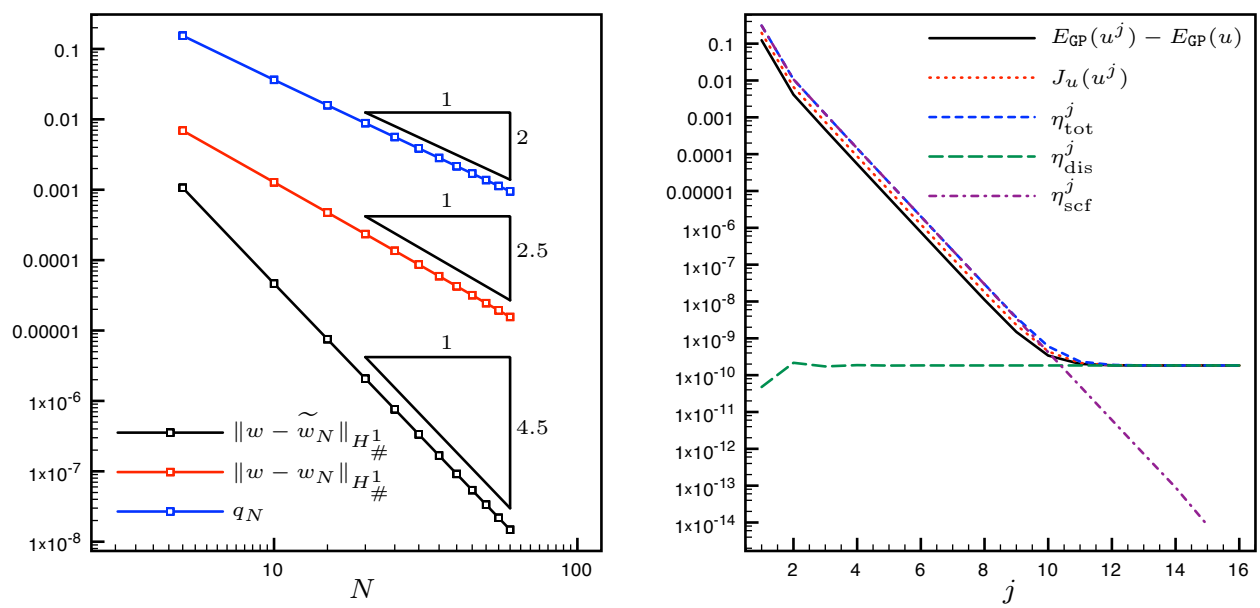

Figure 1. Left: Convergence rate with respect to $N$ with and without post-processing the eigenvector as well as the quotient $q_{N}=\left\|w-\widetilde{w}_{N}\right\|_{H_{\#}^{1}} /\left\|w-w_{N}\right\|_{H_{\#}^{1}}$. Right: Convergence of the error in the energy and the augmented energy difference $J_{u}\left(u^{j}\right)$, and a posteriori estimators with respect to the Roothaan iterations for $N=50$. Gauche : Taux de convergence par rapport à $N$ avec et sans post-traitement du vecteur propre et quotient $q_{N}=\left\|w-\widetilde{w}_{N}\right\|_{H_{\#}^{1}} /\left\|w-w_{N}\right\|_{H_{\#}^{1}}$. Droite : Convergence de l'erreur sur l'énergie et la différence d'énergie augmentée $J_{u}\left(u^{j}\right)$, et estimateurs a posteriori en fonction des itérations de Roothaan pour $N=50$.

\section{References}

[1] R. Becker, C. Johnson, And R. Rannacher, Adaptive error control for multigrid finite element methods, Computing, 55 (1995), pp. 271-288.

[2] E. Cancìs, R. Chakir, And Y. Maday, Numerical analysis of nonlinear eigenvalue problems, J. Sci. Comput., 45 (2010), pp. 90-117.

[3] E. Cancès, G. Dusson, Y. Maday, B. Stamm, and M. Vohralík, On the use of the perturbation method in planewave numerical simulations of linear and nonlinear Schrödinger equations, Full article in preparation.

[4] A. L. Chaillou And M. Suri, A posteriori estimation of the linearization error for strongly monotone nonlinear operators, J. Comput. Appl. Math., 205 (2007), pp. 72-87.

[5] A. ERn AND M. VohralíK, Adaptive inexact Newton methods with a posteriori stopping criteria for nonlinear diffusion PDEs, SIAM J. Sci. Comput., 35 (2013), pp. A1761-A1791. 